\title{
Correlation and dimensional effects of trions in carbon nanotubes
}

\author{
Troels F. Rønnow* and Thomas G. Pedersent \\ Department of Physics and Nanotechnology, \\ Aalborg University, Skjernvej 4A, 9220 Aalborg Øst, Denmark \\ Horia D. Cornean $\ddagger$ \\ Department of Mathematical Sciences, Aalborg University, \\ Frederik Bajers Vej 7G, 9220 Aalborg Øst, Denmark
}

\begin{abstract}
We study the binding energies of singlet trions, i.e. charged excitons, in carbon nanotubes. The problem is modeled, through the effective-mass model, as a three-particle complex on the surface of a cylinder, which we investigate using both one- and two-dimensional expansions of the wave function. The effects of dimensionality and correlation are studied in detail. We find that the Hartree-Fock approximation significantly underestimates the trion binding energy. Combined with band structures calculated using a non-orthogonal nearest neighbour tight binding model, the results from the cylinder model are used to compute physical binding energies for a wide selection of carbon nanotubes. In addition, the dependence on dielectric screening is examined. Our findings indicate that trions are detectable at room temperature in carbon nanotubes with radius below $8 \AA$.
\end{abstract}




\section{INTRODUCTION}

Many applications of semiconducting carbon nanotubes (CNTs) rely on the formation of junctions using either doped $\stackrel{1}{\underline{1}}$ or asymmetrically gated $\mathrm{CNTs}^{2}$. Such junctions are the basis for e.g. CNT based light emitting diodes $\underline{1} \underline{\underline{3}}, \underline{4}$ and other optoelectronic devices. In the description of the optical properties of CNTs, excitons are known to significantly influence emission $^{5,6}$ and absorption ${ }^{7}$. The importance of excitons in CNTs is a consequence of the extremely large binding energy of several hundred meV $\underline{\underline{5}-\underline{\underline{7}}}$. Thus, in an optically excited junction with injected carriers, an exciton in combination with either a hole or an electron may form a three-particle complex known as a trion. It has been shown experimentally that trions form in biased GaAs quantum wells ${ }^{8}$ under optical excitation via the interaction between excitons and injected electrons and holes. Consequently, similar processes are expected to occur in CNTs. Hence, if the binding energy is sufficiently large, these complexes are expected to be of importance for optoelectronic devices and could even be detectable at room temperature.

In traditional semiconductors, trion binding energies and optical spectra have been studied in one ${ }^{9.10}$, two $\frac{11-15}{15}$ and three dimensions ${ }^{15}$. In contrast, trions in CNTs have only been studied in an effectively one-dimensional model ${ }^{16}$, in which carriers were assumed to be completely delocalised around the circumference of the tube. Within this model, it was found that singlet and triplet trions in CNTs are stable against dissociation into excitons and free carriers. Furthermore, the difference in energy between the positive $S^{+}$and negative $S^{-}$ singlet trion states was rather small for mass fractions $\sigma \equiv m_{e} / m_{h} \in[0.8 ; 1]$, where $m_{e}$ is the effective electron mass and $m_{h}$ is the effective hole mass. Also, numerical trion binding energies were estimated for CNTs with radius from $2.5 \AA$ to $10 \AA$. It is clear, however, that a one-dimensional model may have limited applicability for large diameter CNTs, in which motion around the circumference is an important degree of freedom.

The one-dimensional model used in Ref. 16 has many potential applications as it greatly simplifies the complexity of the calculations for trions, biexcitons, charged biexcitons, etc. However, it still remains to assess the quality of this approximation and, therefore, it is important to compare one- and two-dimensional solutions for trions. It is clear that the 
effective-mass model used in Ref. 16 is an approximation to more accurate ab initio approaches. However, comparison with ab initio results for the exciton energy and its scaling with tube radius ${ }^{17}$ as well as the exciton wave function 18 demonstrates excellent agreement between these different approaches 17 , 18. In this paper, we investigate two simplifications of the full effective-mass trion problem: First, the one-dimensional model is examined by comparison to two-dimensional solutions of the full problem. Second, the accuracy of the Hartree-Fock approximation for trion binding energies is determined in both one- and twodimensional models. As our starting point for both models, we expand the wave function in a convenient basis. The same basis expansion is applied in the Hartree-Fock calculation, which is briefly outlined. Next, we investigate the binding energies of trions, in both the one- and two-dimensional models, as functions of cylinder radius $r$ and mass fraction $\sigma$. We compare the Hartree-Fock solutions with the full solutions (at $\sigma=0.0$ ), and we analyze the distribution of electrons along the circumference in a negative trion state $S^{-}$for the two methods. Finally, the CNT band structure is used to convert model results into physical binding energies for a wide range of CNTs, using both the one- and two-dimensional models.

\section{THEORY}

Adopting the fundamental trion equation from Ref. 15 and introducing relative cylindrical coordinates along with removal of center-of-mass motion, we get the Hamiltonian for the negative trion

$$
\hat{H}_{-}=\hat{h}_{1}+\hat{h}_{2}-\frac{2 \sigma}{1+\sigma}\left(\frac{\partial^{2}}{\partial x_{1} \partial x_{2}}+\frac{1}{r^{2}} \frac{\partial^{2}}{\partial \theta_{1} \partial \theta_{2}}\right)+V\left(x_{1}-x_{2}, \theta_{1}-\theta_{2}\right),
$$

where $r$ is the nanotube radius and $x_{i}$ and $r \theta_{i}$ denote axial and circumference coordinates, respectively, as shown in Fig. 1. The exciton operators, $\hat{h}_{1}$ and $\hat{h}_{2}$, are given by

$$
\hat{h}_{i}=-\frac{\partial^{2}}{\partial x_{i}^{2}}-\frac{1}{r^{2}} \frac{\partial^{2}}{\partial \theta_{i}^{2}}-V\left(x_{i}, \theta_{i}\right),
$$

and the Coulomb potential in the cylinder geometry is given as $\frac{19}{9}$

$$
V(x, \theta)=\frac{2}{\sqrt{x^{2}+4 r^{2} \sin ^{2} \frac{\theta}{2}}} .
$$

Here, the coordinate pairs $\left(x_{1}, \theta_{1}\right)$ and $\left(x_{2}, \theta_{2}\right)$ describe the motion of the first and second

electron relative to the hole, respectively. The positive trion Hamiltonian $\hat{H}_{+}$is found by 


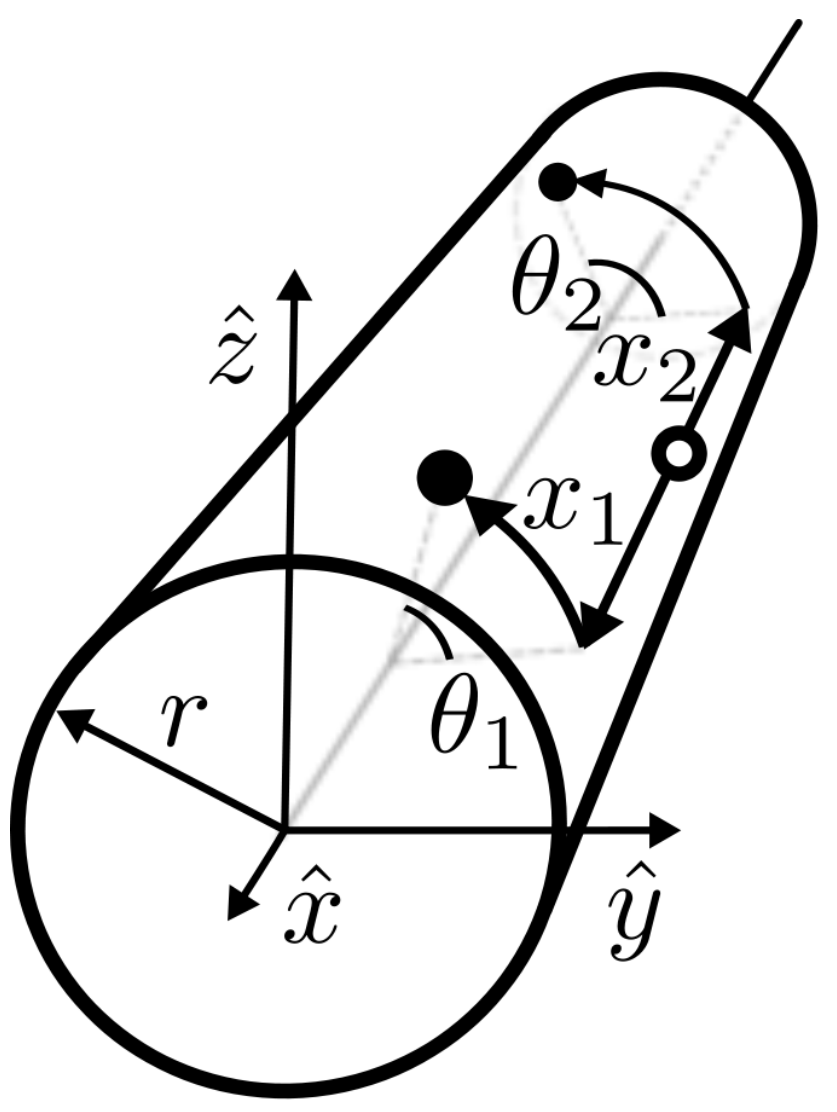

FIG. 1. Geometry of a negative trion on the surface of a cylinder of radius $r$, described in relative coordinates. The filled circles illustrate electrons and the open circle illustrates a hole.

the replacement $\sigma \rightarrow \sigma^{-1}$. Note that $\hat{H}_{-}$and $\hat{H}_{+}$have incorrectly been interchanged in Ref. 16. The Hamiltonian is expressed in natural exciton units, i.e. effective Bohr radii $a_{B}^{*}$ and effective Rydbergs $R y^{*}$ for distances and energies, respectively.

If the radius $r$ is much smaller than the effective Bohr radius $a_{B}^{*}$, the electrons are highly delocalised around the circumference and the angular dependence of the wave function for the lowest states will be nearly constant. In contrast, when $r$ is comparable to $a_{B}^{*}$, the angular dependence is expected to have a cusp-like behaviour similar to the one found for excitons $\frac{19}{}$. A function that suitably describes this behaviour is the absolute value of sine $\left|\sin \frac{\theta}{2}\right|$. Thus, it would be reasonable to model the angular part of the wave function as a linear combination of the two limits. Moreover, to model the electron-electron repulsion for $\hat{H}_{-}$and hole-hole repulsion for $\hat{H}_{+}$, the function $\left|\sin \left(\frac{\theta_{1}-\theta_{2}}{2}\right)\right|$ constitutes a sensible choice as it attains its minimum along the diagonal $\theta_{1}=\theta_{2}$. Having this in mind, we expand the wave 
function according to

$$
\psi\left(x_{1}, \theta_{1}, x_{2}, \theta_{2}\right)=\sum_{i, j, k}^{N_{1}, N_{2}, N_{3}} \sum_{l}^{4} c_{i j k l} \phi_{i}\left(x_{1}\right) \phi_{j}\left(x_{2}\right) \phi_{k}\left(x_{1}-x_{2}\right) \varphi_{l}\left(\theta_{1}, \theta_{2}\right),
$$

with Gaussians $\phi_{i}(x)=e^{-\alpha_{i} x^{2}}$ along the translational direction and an angular basis $\left\{\phi_{l}\right\}_{l=1}^{4}$ along the circumference, where

$$
\varphi_{l}\left(\theta_{1}, \theta_{2}\right)=\frac{1}{2 \pi}\left\{\begin{array}{cl}
1 & l=1, \\
\left|\sin \frac{\theta_{1}}{2}\right| & l=2, \\
\left|\sin \frac{\theta_{2}}{2}\right| & l=3, \\
\left|\sin \frac{\theta_{1}-\theta_{2}}{2}\right| & l=4 .
\end{array}\right.
$$

Here, $i, j$ and $k$ run from 1 to $N_{1}, N_{2}$ and $N_{3}$, respectively, and $N=N_{1} \cdot N_{2} \cdot N_{3}$ is the total number of Gaussians used in the expansion. The expansion coefficients $c_{i j k l}$ are obtained from the eigenvector $\vec{c}$ of a matrix equation of the form $(\overleftrightarrow{K}+\overleftrightarrow{U}) \cdot \vec{c}=E_{T} \overleftrightarrow{S} \cdot \vec{c}$. We define the trion binding energy $E_{B} \equiv E_{X}-E_{T}$ as the difference between the exciton binding energy $E_{X}$ and the trion energy $E_{T}$, where a positive $E_{B}$ indicates a stable trion. The kinetic matrix $\overleftrightarrow{K}$ and overlap matrix $\overleftrightarrow{S}$ elements turn out as $S_{i j k l, i^{\prime} j^{\prime} k^{\prime} l^{\prime}}=S_{i j k, i^{\prime} j^{\prime} k^{\prime}}^{(T)} S_{l l^{\prime}}^{(C)}$ and

$$
\begin{aligned}
K_{i j k l, i^{\prime} j^{\prime} k^{\prime} l^{\prime}}=S_{i j k, i^{\prime} j^{\prime} k^{\prime}}^{(T)}\left(K_{l l^{\prime}}^{(C)}+\frac{2 \sigma}{1+\sigma}\right. & \left.K_{l l^{\prime}}^{(C M)}\right) \\
& +\left(K_{i j k, i^{\prime} j^{\prime} k^{\prime}}^{(T)}+K_{j i k, j^{\prime} i^{\prime} k^{\prime}}^{(T)}+\frac{2 \sigma}{1+\sigma} K_{i j k, i^{\prime} j^{\prime} k^{\prime}}^{(T M)}\right) S_{l l^{\prime}}^{(C)}
\end{aligned}
$$

with

$$
\begin{aligned}
S_{i j k, i^{\prime} j^{\prime} k^{\prime}}^{(T)} & =\frac{\pi}{\sqrt{\left(\alpha_{i}+\alpha_{i^{\prime}}\right)\left(\alpha_{j}+\alpha_{j^{\prime}}\right)\left(\alpha_{k}+\alpha_{k^{\prime}}\right)}}, \\
K_{i j k, i^{\prime} j^{\prime} k^{\prime}}^{(T)} & =\frac{2 \pi\left(\alpha_{k^{\prime}} \alpha_{k}\left(\alpha_{j}+\alpha_{j^{\prime}}+\alpha_{i}+\alpha_{i^{\prime}}\right)+\alpha_{i^{\prime}} \alpha_{i}\left(\alpha_{k^{\prime}}+\alpha_{k}\right)\right)}{\left(\left(\alpha_{j}+\alpha_{j^{\prime}}\right)\left(\alpha_{i}+\alpha_{i^{\prime}}+\alpha_{k}+\alpha_{k^{\prime}}\right)+\left(\alpha_{i}+\alpha_{i^{\prime}}\right)\left(\alpha_{k}+\alpha_{k^{\prime}}\right)\right)^{3 / 2}} \\
& +\frac{2 \pi\left(\alpha_{j}+\alpha_{j^{\prime}}\right)\left(\alpha_{k^{\prime}} \alpha_{i}+\alpha_{i^{\prime}} \alpha_{k}+\alpha_{i} \alpha_{i^{\prime}}\right)}{\left(\left(\alpha_{j}+\alpha_{j^{\prime}}\right)\left(\alpha_{i}+\alpha_{i^{\prime}}+\alpha_{k}+\alpha_{k^{\prime}}\right)+\left(\alpha_{i}+\alpha_{i^{\prime}}\right)\left(\alpha_{k}+\alpha_{k^{\prime}}\right)\right)^{3 / 2}}, \\
K_{l l^{\prime}}^{(C)} & =\frac{1}{8}\left(1-\delta_{1 l}\right) \delta_{l l^{\prime}}, \\
K_{i j k, i^{\prime} j^{\prime} k^{\prime}}^{(T M)} & =-\frac{2 \pi\left(\alpha_{k} \alpha_{k^{\prime}}\left(\alpha_{i}+\alpha_{i^{\prime}}+\alpha_{j}+\alpha_{j^{\prime}}\right)+\alpha_{i} \alpha_{j} \alpha_{k^{\prime}}+\alpha_{i^{\prime}} \alpha_{j^{\prime}} \alpha_{k}\right)}{\left(\left(\alpha_{i}+\alpha_{i^{\prime}}\right)\left(\alpha_{j}+\alpha_{j^{\prime}}+\alpha_{k}+\alpha_{k^{\prime}}\right)+\left(\alpha_{j}+\alpha_{j^{\prime}}\right)\left(\alpha_{k}+\alpha_{k^{\prime}}\right)\right)^{3 / 2}}, \\
K_{l l^{\prime}}^{(C M)} & =-\frac{1}{8} \delta_{4 l} \delta_{l l^{\prime}},
\end{aligned}
$$

where $\delta_{i j}$ is the Kronecker delta and with the angular overlap integrals $S_{l l^{\prime}}^{(C)}$ given in Tab. I. In the derivation, care should be taken as the second derivative of the absolute value 


\begin{tabular}{c|cccc}
$S_{l l^{\prime}}^{(C)}$ & 1 & 2 & 3 & 4 \\
\hline 1 & 1 & $\frac{2}{\pi}$ & $\frac{2}{\pi}$ & $\frac{2}{\pi}$ \\
2 & $\frac{2}{\pi}$ & $\frac{1}{2}$ & $\frac{4}{\pi^{2}}$ & $\frac{4}{\pi^{2}}$ \\
3 & $\frac{2}{\pi}$ & $\frac{4}{\pi^{2}}$ & $\frac{1}{2}$ & $\frac{4}{\pi^{2}}$ \\
4 & $\frac{2}{\pi}$ & $\frac{4}{\pi^{2}}$ & $\frac{4}{\pi^{2}}$ & $\frac{1}{2}$
\end{tabular}

TABLE I. Table of angular overlap integrals.

of a sine gives a delta function, which takes many of the kinetic energy elements to zero. The potential energy matrix elements turn out to be slightly more complicated and can be expressed in terms of 4 different Meijer $G$ functions.

In order to formulate the problem in terms of the Hartree-Fock approximation we now assume that the eigenfunction of the operator Eq. (1) is a Slater determinant of single electron states $\chi(x, \theta)$ with anti-symmetry in the spin part. From standard quantum mechanics ${ }^{20}$ we then obtain the Fock operator for the corresponding system

$$
\hat{F}=-\frac{\partial^{2}}{\partial x^{2}}-\frac{1}{r^{2}} \frac{\partial^{2}}{\partial \theta^{2}}-\frac{2}{\sqrt{x^{2}+4 r^{2} \sin ^{2} \frac{\theta}{2}}}+V_{H}(x, \theta),
$$

where the Hartree potential $V_{H}$ is given by

$$
V_{H}(x, \theta)=2 \int_{-\infty}^{\infty} d x^{\prime} \int_{-\pi}^{\pi} d \theta^{\prime} \frac{\left|\chi\left(x^{\prime}, \theta^{\prime}\right)\right|^{2}}{\sqrt{\left(x-x^{\prime}\right)^{2}+4 r^{2} \sin ^{2} \frac{\theta-\theta^{\prime}}{2}}}
$$

From the eigenvalue equation $\hat{F} \chi(x, \theta)=\varepsilon \chi(x, \theta)$ the Hartree-Fock eigenvalues are found and the lowest one $\varepsilon_{0}$ is related to the trion ground state energy in the usual way

$$
E_{T}^{(H F)}=2 \varepsilon_{0}-\int_{-\infty}^{\infty} d x \int_{-\pi}^{\pi} d \theta|\chi(x, \theta)|^{2} V_{H}(x, \theta) .
$$

Expanding $\chi(x, \theta)$ in a basis of Gaussians along the translational direction and absolute value of sines along the circumference, as done for the full problem, we are capable of solving the Hartree-Fock eigenvalue equation self-consistently.

\section{RESULTS AND DISCUSSION}

Optimisation of the Gaussian coefficients was carried out using a steepest descent method. The following coefficients, in units of $a_{B}^{*-2}$, were found for the different optimisations: $\alpha_{i} \in\{0.143,1.16,4.98,29.0,250\}$ for both the 1D and 2D exciton and $\alpha_{i}, \alpha_{j}, \alpha_{k} \in$ 


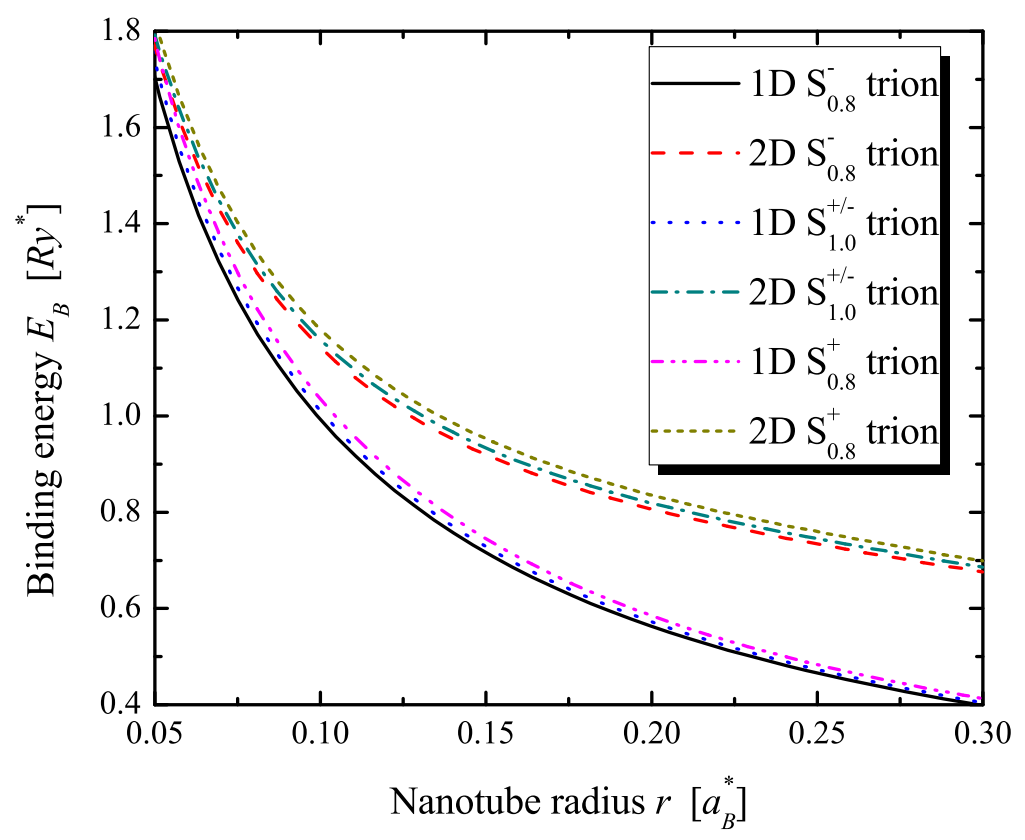

FIG. 2. Comparison between the one- and two-dimensional models. For each of the two models three cases are shown, namely, $S_{0.8}^{+}, S_{1.0}^{+/-}$and $S_{0.8}^{-}$. The positive and negative trion binding energies were calculated for $\sigma=0.80$. The $S_{1.0}^{+/-}$binding energies were calculated for $\sigma=1.0$.

$\{0.0651,0.145,1.68,9.65,48.7\}$ for the 1D trion. For the 2D trion, the Gaussian coefficients were found to be $\alpha_{i}, \alpha_{j} \in\{0.165,1.68,9.65,48.7\}$ for the functions $e^{-\alpha_{i} x^{2}}$ and $e^{-\alpha_{j} y^{2}}$, and $\alpha_{k} \in\{0.0000171,1.68,9.98,48.7\}$ for the functions $e^{-\alpha_{k}(x-y)^{2}}$. Similarly, the coefficients for the Hartree-Fock calculations were found to be $\alpha_{i}, \alpha_{j} \in\{0.0648,0.195,1.04,5.28,27.5,99.3,250\}$ for both the $1 \mathrm{D}$ and $2 \mathrm{D}$ cases. All optimisations were carried out at $r_{0}=0.1 a_{B}^{*}$ and all coefficients were subsequently scaled with $r_{0}^{2} / r^{2}$ for calculations involving other radii.

The binding energies $E_{B}$ of trions were found as functions of radius for both the oneand two-dimensional models. In Fig. 2, it is seen that the one- and two-dimensional results deviate less as $r$ approaches zero, as expected. It is important to realize that the effective Bohr radius $a_{B}^{*}$ is dependent on nanotube species via the effective masses and dielectric constant $\varepsilon$. Due to the scaling of masses with nanotube radius, it turns out that $r$ is, in fact, roughly proportional to $a_{B}^{*}$. For CNTs embedded in a medium with $\varepsilon=3.5$ it is found that $\frac{19}{9}$ $r \approx 0.1 a_{B}^{*}$, and for this value the correction is roughly $13 \%$ of the two-dimensional result. 


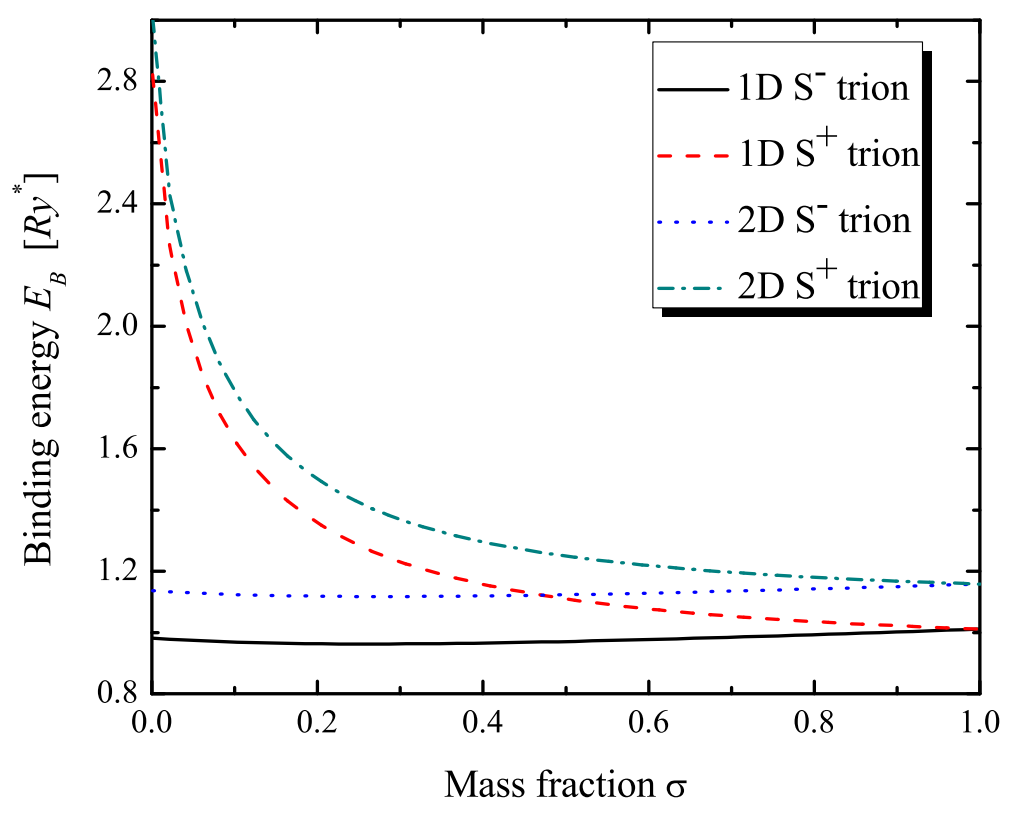

FIG. 3. Trion binding energy as a function of the mass fraction $\sigma=m_{e} / m_{h}$ for the one- and two-dimensional models. The calculations were done for $r=0.1 a_{B}^{*}$.

The one-dimensional model performs significantly worse when the radius is increased. For $r \approx 0.3 a_{B}^{*}$ the one-dimensional result is $42 \%$ below the two-dimensional one and, as would be expected, the one-dimensional model can only be considered a good approximation for $r$ close to zero. Realistically, though, $r>0.2 a_{B}^{*}$ will only occur for CNTs embedded in media with small screening $\varepsilon<1.75$.

From Eq. (1) it follows that the mass fraction $\sigma$ affects the binding energy via a mixed kinetic energy term coupling the two relative coordinates $\left(x_{1}, \theta_{1}\right)$ and $\left(x_{2}, \theta_{2}\right)$. The mass fraction $\sigma$ of CNTs varies between 0.86 and $1.0^{16}$ and to gain an idea of the upper and lower bounds of the trion binding energies we have plotted the positive and negative trions at $\sigma=0.8$ in Fig. 2. Actual binding energies should lie between the $S_{0.8}^{-}$and $S_{1.0}^{+/-}$energy curves and the $S_{1.0}^{+/-}$and $S_{0.8}^{+}$curves for the negative and positive trion, respectively. It is seen that the difference is fairly small for both the one- and two-dimensional models. In Fig. 3. we illustrate the binding energies as functions of the mass fraction $\sigma$ for both models for $r=0.1 a_{B}^{*}$. In both cases, the binding energy for the negative trion $S^{-}$was found to have 


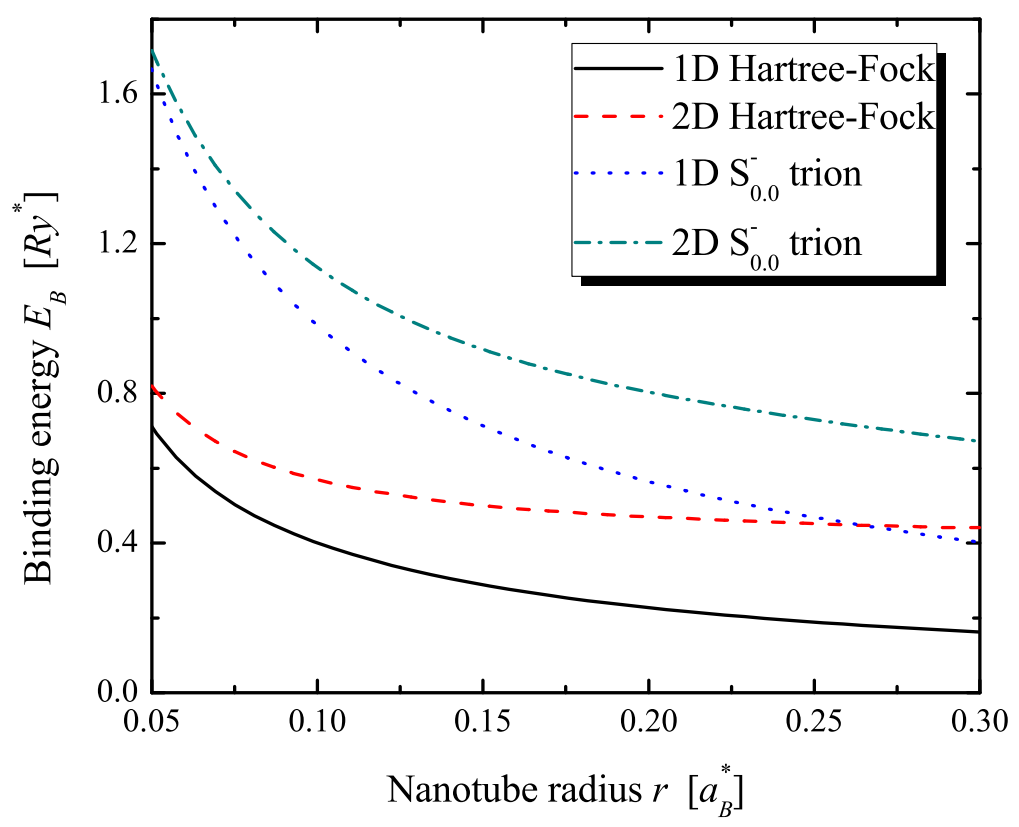

FIG. 4. Solutions for the one- and two-dimensional models compared with the corresponding Hartree-Fock solutions. The calculations were carried out at $\sigma=0.0$.

little variation for $\sigma \in[0 ; 1]$. As a consequence, the results calculated for $S_{0.0}^{-}$are, in fact, excellent approximations for both $S_{\sigma}^{+}$and $S_{\sigma}^{-}$with mass fractions in the range $\sigma \in[0.8 ; 1]$. It follows that neglecting the mixed kinetic energy term is an acceptable approximation, which in the worst case would give an error of $3.2 \%$ at $r=0.1 a_{B}^{*}$.

Next we calculated the binding energies using the Hartree-Fock approximation. As demonstrated above, the mixed kinetic energy term is only a minor correction for actual nanotubes. Thus, in the following we ignore this term and limit the discussion to $S_{0.0}^{-}$states. In Fig. 4, the Hartree-Fock energies are plotted along with the $S_{0.0}^{-}$trion energies for both one- and two-dimensional solutions. The Hartree-Fock solutions significantly underestimate trion binding energies is both cases. Differences up to a factor of 2 , in the two-dimensional case, were found. The best Hartree-Fock result obtained in the interval $r \in[0 ; 0.3]$ was at the end point $r=0.3 a_{B}^{*}$ where the Hartree-Fock energy is $60 \%$ of the correct result. The trion problem of two electrons interacting with a positive hole is mathematically similar to the He atom. Hence, the dramatic failure of the Hartree-Fock approximation is surprising 
at first sight. However, the failure is readily ascribed to several characteristic factors in the trion case. Primarily, we focus here on the binding energy $E_{B}=E_{X}-E_{T}$ given as the difference between exciton and trion energies. Hence, relatively small errors in the HartreeFock trion energies $E_{T}^{(H F)}$ lead to large errors in the binding energies provided $E_{T}^{(H F)}-E_{T}$ is comparable to $E_{X}-E_{T}$. Secondly, Fig. [ 4 shows that the error increases as we decrease the radius, i.e. when the effective dimension of the system decreases from two towards unity. Actual CNTs can be regarded as effectively 1.7 dimensional systems ${ }^{21}$. The approximate treatment of electron-electron repulsion in the Hartree-Fock calculation is more serious in a low-dimensional geometry due to the increased overlap between electrons enforced by the geometry. Hence, the Hartree-Fock error for the (three dimensional) He atom is small even if the energy is measured relative to the hydrogen atom. Finally, in the present problem, the strengths (charges) of the electron-hole and electron-electron are identical. In He, the larger nuclear charge $Z=2$ enhances the electron-nucleus interaction. As a result, the contribution to the energy from the electron-electron repulsion is relatively smaller and, thus, the Hartree-Fock approximation is less inaccurate.

To investigate the behaviour of the wave function around the circumference the probability distribution, averaged over the translational coordinates $x_{1}$ and $x_{2}$, as a function of angles,

$$
\begin{aligned}
P_{T}\left(\theta_{1}, \theta_{2}\right) & =\int_{-\infty}^{\infty} \int_{-\infty}^{\infty} d x_{1} d x_{2}\left|\psi\left(x_{1}, \theta_{1}, x_{2}, \theta_{2}\right)\right|^{2} \\
& =\sum_{l, l^{\prime}}^{4} \sum_{i, j, k}^{4} \sum_{i^{\prime}, j^{\prime}, k^{\prime}}^{4} S_{i j k, i^{\prime} j^{\prime} k^{\prime}}^{(T)} c_{i j k l} c_{i^{\prime} j^{\prime} k^{\prime} l^{\prime}} \varphi_{l}\left(\theta_{1}, \theta_{2}\right) \varphi_{l^{\prime}}\left(\theta_{1}, \theta_{2}\right),
\end{aligned}
$$

has been shown in the left column of Fig. 沾a-c for $r=0.05 a_{B}^{*}, r=0.10 a_{B}^{*}$ and $r=0.25 a_{B}^{*}$. The probability distribution gives the probability $P_{T}\left(\theta_{1}, \theta_{2}\right) d \theta_{1} d \theta_{2}$ of finding the electrons with angles between $\theta_{1}$ and $\theta_{1}+d \theta_{1}$, and, $\theta_{2}$ and $\theta_{2}+d \theta_{2}$ from the hole, respectively. It is normalised such that integration over both angles from $-\pi$ to $\pi$ yields 1 . It is seen that the probability distribution tends to be more delocalised as $r$ tends to zero. This is also what we would expect since a purely one-dimensional system has no angular dependence. Further it is noticed that the distribution has a butterfly shape as is seen in other cases $\frac{16}{22}$. In Fig. $5 \mathrm{~d}$ we have plotted the exciton probability distribution as function of the angle for $r$ between 
$0.05 a_{B}^{*}$ and $r=0.25 a_{B}^{*}$. The exciton probability distribution is given by

$$
P_{X}(\theta)=\int_{-\infty}^{\infty} d x\left|\psi_{X}(x, \theta)\right|^{2}=\sum_{j, j^{\prime}}^{2} \sum_{i, i^{\prime}}^{5} c_{i j} c_{i^{\prime} j^{\prime}} \sqrt{\frac{\pi}{\alpha_{i}+\alpha_{i^{\prime}}}} \varphi_{j}^{(X)}(\theta) \varphi_{j^{\prime}}^{(X)}(\theta),
$$

where $\varphi_{1}^{(X)}(\theta)=1 / \sqrt{2 \pi r}$ and $\varphi_{2}^{(X)}(\theta)=\left|\sin \frac{\theta}{2}\right| / \sqrt{2 \pi r}$. This plot confirms the contracting behaviour of the trion probability distribution seen in a-c. In Fig. 5e, the Hartree-Fock probability distribution has been plotted for $r=0.10 a_{B}^{*}$. Clearly, the effects of approximating the electron-electron repulsion are largest along the diagonal $\theta_{1}=\theta_{2}$. This is emphasised in the difference plot Fig. 5f, where the difference between the Hartree-Fock distribution and the full distribution has been plotted in percent. The errors in the Hartree-Fock distribution vary from $-2 \%$ up to $4 \%$. The delocalization of the trion wave function is enhanced by the nearly equal electron and hole masses. Hence, the centre of mass will not coincide with the position of any of the constituents but, rather, lie somewhere in between. This is another notable difference between trions and He atoms, for which the centre of mass will practically fall on top of the nucleus. As a consequence, both electrons and holes are almost completely delocalized around the circumference in the realistic trion case $\sigma \approx 1.0$ and $r \approx 0.1 a_{B}^{*}$, as illustrated in Fig. 5b.

Whether trions in CNTs will be detectable at room temperature is determined by the magnitude of the trion binding energy $E_{B}$ relative to the thermal energy $k_{B} T$. The trion binding energy depends on the static dielectric constant $\varepsilon$ as well as the reduced electronhole pair mass $\mu$, through the effective Rydberg $R y^{*}=13.6 \mathrm{eV} \cdot \mu / \varepsilon^{2}$ and the effective Bohr radius $a_{B}^{*}=0.529 \AA \cdot \varepsilon / \mu^{16,19}$. The reduced mass $\mu$ is obtained from the CNT band structure, and in order to determine the hole and electron masses in CNTs, for a particular chiral index $(n, m)$, we used a non-orthogonal nearest neighbour tight binding model. The transfer integral was chosen $\operatorname{as}^{23} t=-2.89 \mathrm{eV}$ and the overlap as $s=0.1$. The soundness of these parameters follows from the fact that the predicted Fermi velocity of graphene $v_{F}=9.6 \cdot 10^{5} \mathrm{~m} / \mathrm{s}$ is within $5.7 \%$ of the average experimental value ${ }^{24}$. Since the CNT band structures are derived from the graphene band structure this indicates that effective hole and electron masses will be estimated with a similar error. Using this tight binding model the reduced mass was found as $\mu=1 /\left(m_{h}^{-1}+m_{e}^{-1}\right)$. The dielectric constant $\varepsilon$ on the other hand, is determined partly by the CNTs and partly by their surroundings. It has been shown in several articles ${ }^{25}-27$ that the contribution from the surroundings plays a significant role in the determination of optical transition energy in CNTs. Further, the electro-static potential 


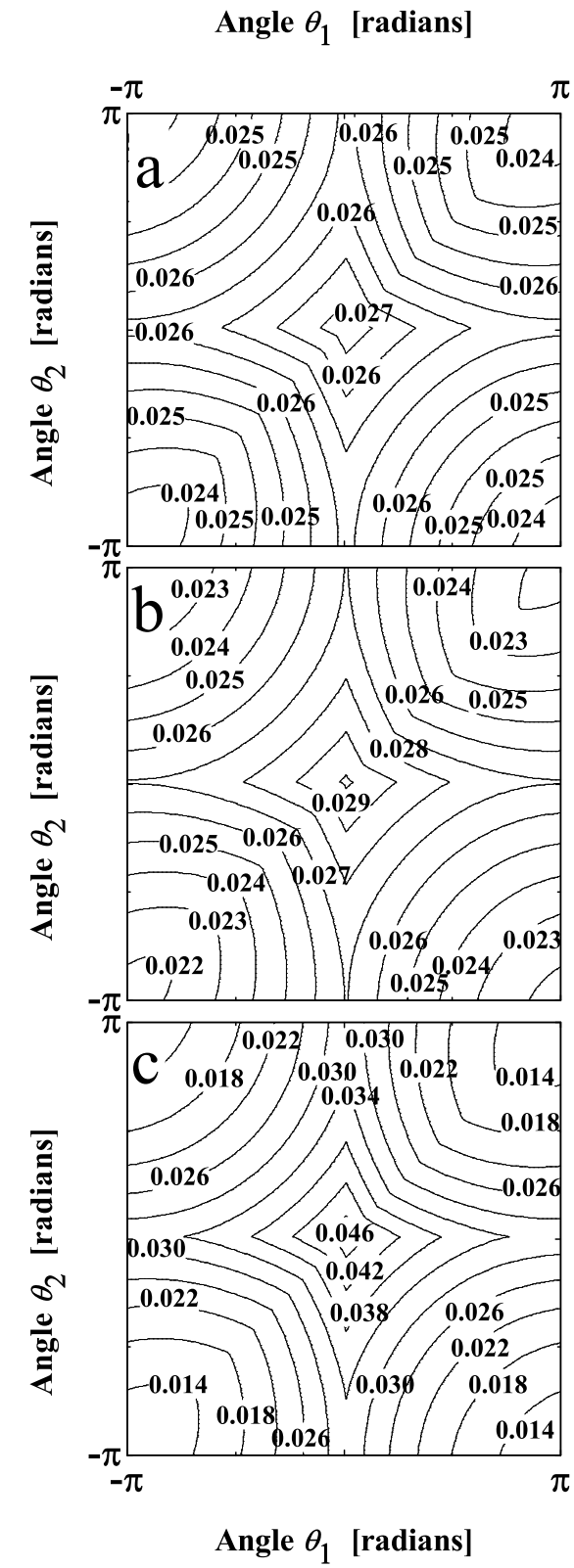

$\pi$
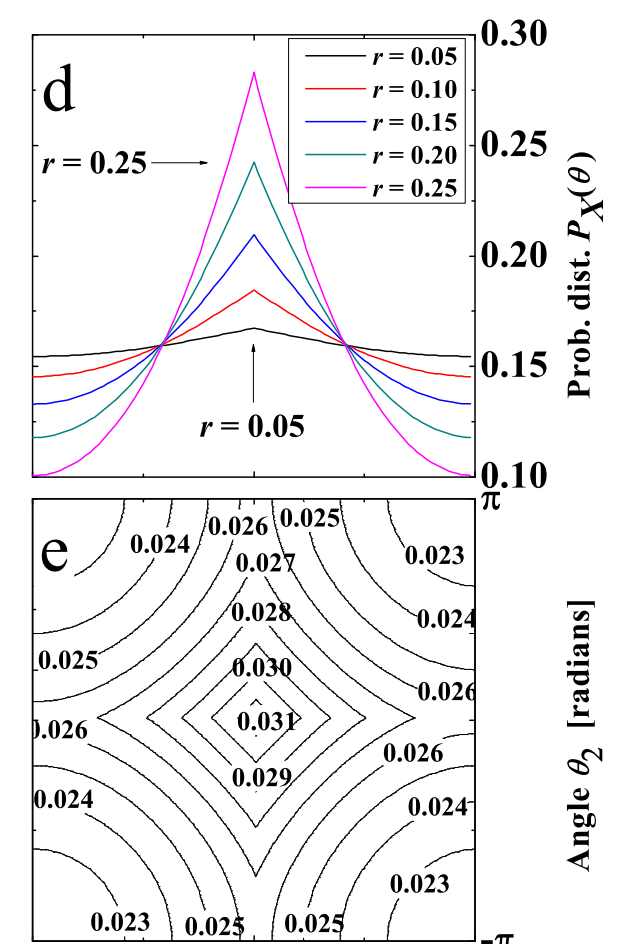

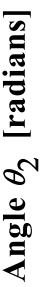

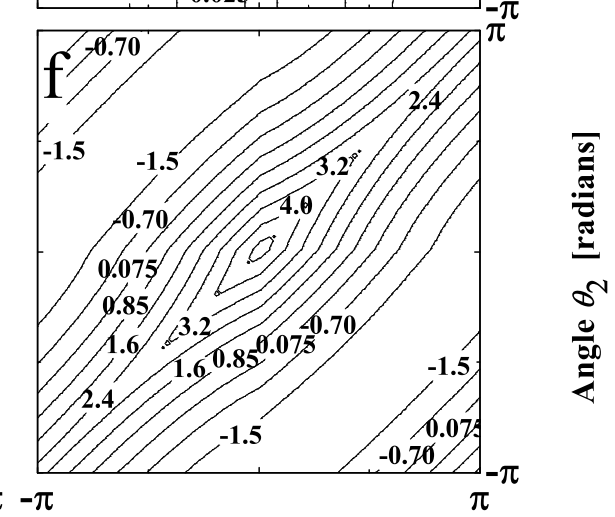

Angle $\theta_{1}$ [radians]

FIG. 5. Probability distributions as a functions of angles for (a) $r=0.05 a_{B}^{*}$, (b) $r=0.10 a_{B}^{*}$ and (c) $r=0.25 a_{B}^{*}$ for the singlet trion $S_{1.0}^{+/-}$calculated with a basis expansion. The exciton probability distributions have been shown in (d) for the radius varying from $r=0.05 a_{B}^{*}$ to $r=0.25 a_{B}^{*}$. In (e), the Hartree-Fock probability distribution is shown at $r=0.1 a_{B}^{*}$ and (f) shows percent wise difference between the Hartree-Fock solution and the solution obtained from the full problem at $r=0.1 a_{B}^{*}$ 
$\phi$, in an quasi one-dimensional nano structure confined along $y$ and $z$, will be governed by the perpendicular component $\varepsilon_{\perp}$, since ${ }^{28} \phi \propto\left(\varepsilon_{z} \varepsilon_{y} x^{2}+\varepsilon_{x} \varepsilon_{z} y^{2}+\varepsilon_{x} \varepsilon_{y} z^{2}\right)^{-1 / 2} \approx \varepsilon_{\perp}^{-1}|x|^{-1}$ if the tube radius is small. It has been shown that $\varepsilon_{\perp}$, is small for a wide range of nanotubes in Ref. 29, and therefore the surrounding material is expected to dominate the static dielectric constant $\varepsilon$. Moreover, the results expressed in effective units are universal results and do not depend on $\varepsilon$. The dependence arises only through the conversion into physical units (eV and $\AA$ ) via the effective Rydberg $R y^{*}$ and Bohr radius $a_{B}^{*}$, similarly to the exciton $\underline{\underline{19}}$ and biexciton ${ }^{30}$. Since the dielectric constant depends on the material in which the CNTs are embedded, $a_{B}^{*}$ and $R y^{*}$ should be determined for the individual experiment. Therefore $\varepsilon$ can be regarded as an experimental parameter varying from sample to sample. Well-established methods for synthesising samples with high concentrations of $(6,5)$ CNTs exist $\frac{31}{1}, \underline{32}$ and we will therefore use $(6,5)$ as an example in the following. CNTs having chiral index $(6,5)$ have in some cases been suspended in sodium cholat ${ }^{32}$ and, in other cases, embedded in a polymeric matrix $\underline{\underline{31}}$. The dielectric constant $\varepsilon$ should be chosen according to either of these materials. However, no exact values of $\varepsilon$ were found for the above suspension materials. It has been shown that the photonic transition energy as a function of environmental dielectric constant saturates near $\underline{26} \varepsilon_{e n v}^{(s a t)} \approx 5.0$. Expecting that the surrounding material will have a dielectric constant somewhat above the dielectric constant of air, $\varepsilon=3.5$ is considered as a reasonable average. The radius for $(6,5)$ CNTs is $r=3.73 \AA$ and the effective masses of electron and hole turn out to be $m_{e}=0.0803$ and $m_{h}=0.0866$, respectively, which leads to a reduced mass $\mu=0.0417$. The effective Rydberg and Bohr radius are found to be $R y^{*}=0.0462 \mathrm{eV}$ and $a_{B}^{*}=44.5 \AA$, respectively. With these values the effective radius is $r=0.084 a_{B}^{*}$, and using this, the negative trion binding energy can be calculated to $E_{B}=1.28 R y^{*} \approx 59 \mathrm{meV}$. Hence, for this species $E_{B}$ is larger than $k_{B} T=26 \mathrm{meV}$ and the trion state for $(6,5)$ CNTs is expected to be detectable at room temperature.

In the hope that these results will stimulate measurements of the trion binding, we have calculated the energies at $\varepsilon \in[2.0 ; 5.0]$, which corresponds to finding the binding energy for $(6,5)$ CNTs suspended in a large variety of solutions. We found that the binding energy, in this range for $\varepsilon$, will be at least $E_{B}=36 \mathrm{meV}$ and at most $E_{B}=132 \mathrm{meV}$. The result has 


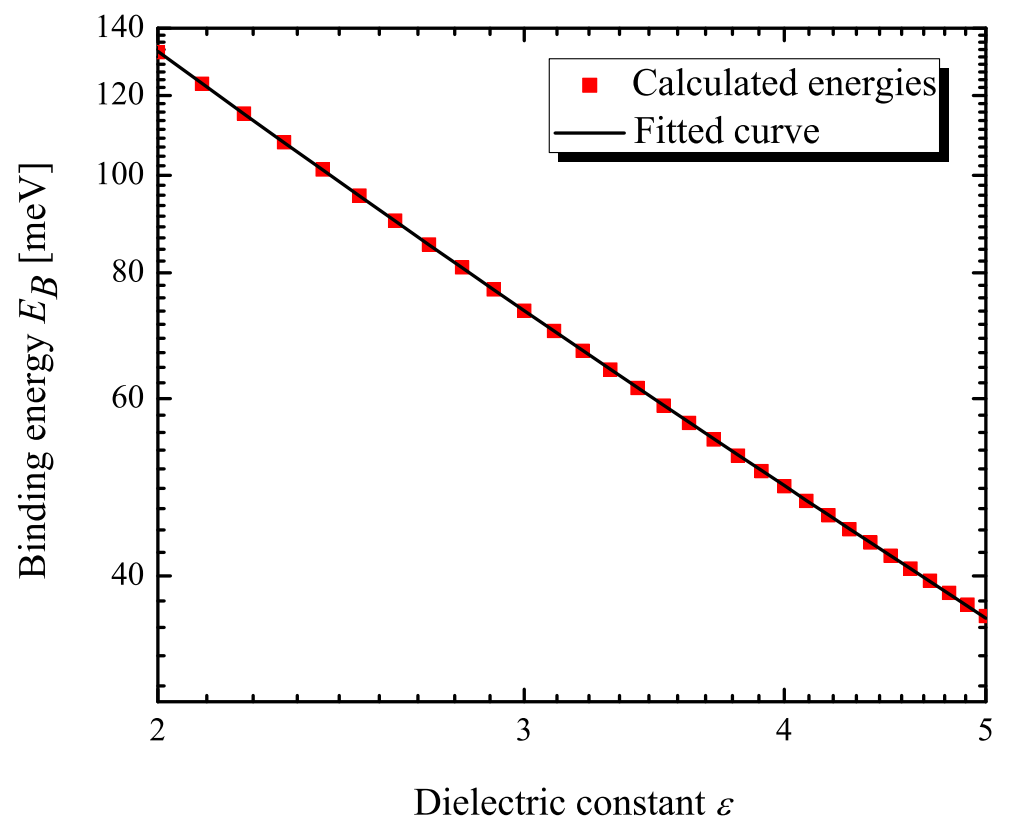

FIG. 6. Binding energy of negative trions in $(6,5)$ CNTs as a function of dielectric constant $\varepsilon$.

been shown in Fig. [6 in a double logarithmic plot and we also include a curve fit given by

$$
E_{B}(\varepsilon) \approx\left(0.372 \varepsilon^{-1.56}+0.00608\right) \mathrm{meV}
$$

The result is not surprising as it simply emphasises the importance of choice of suspension material with respect to measuring the binding energy of trions. As a consequence, experiments should be conducted with suspension materials with low dielectric constants. Also, the power dependence is what would be expected as a similar result was found for the exciton ${ }^{19}$, where it was shown that $E_{X}(r) \propto r^{-0.6} R y^{*}$, with $r$ in units of $a_{B}^{*}$. Using the effective Rydberg and Bohr radius it is easily shown that the exciton energy for CNTs follows a similar power law $E_{X}(\varepsilon) \propto \varepsilon^{-1.4}$.

Keeping $\varepsilon=3.5$ constant, we found the positive and negative trion binding energies for all semiconducting CNTs with $3 \AA \leq r \leq 15 \AA$. The results are seen in Fig. 7. The binding energies were found using both the one- and two-dimensional models. As seen in the inset, the two-dimensional model has improved the energy, compared with the one-dimensional one, with up to $15 \%$, and on average $11 \%$. It is noticed that in order to observe trions in CNTs at room temperature, the radius of the CNTs must be below approximately $8 \AA$. Since 


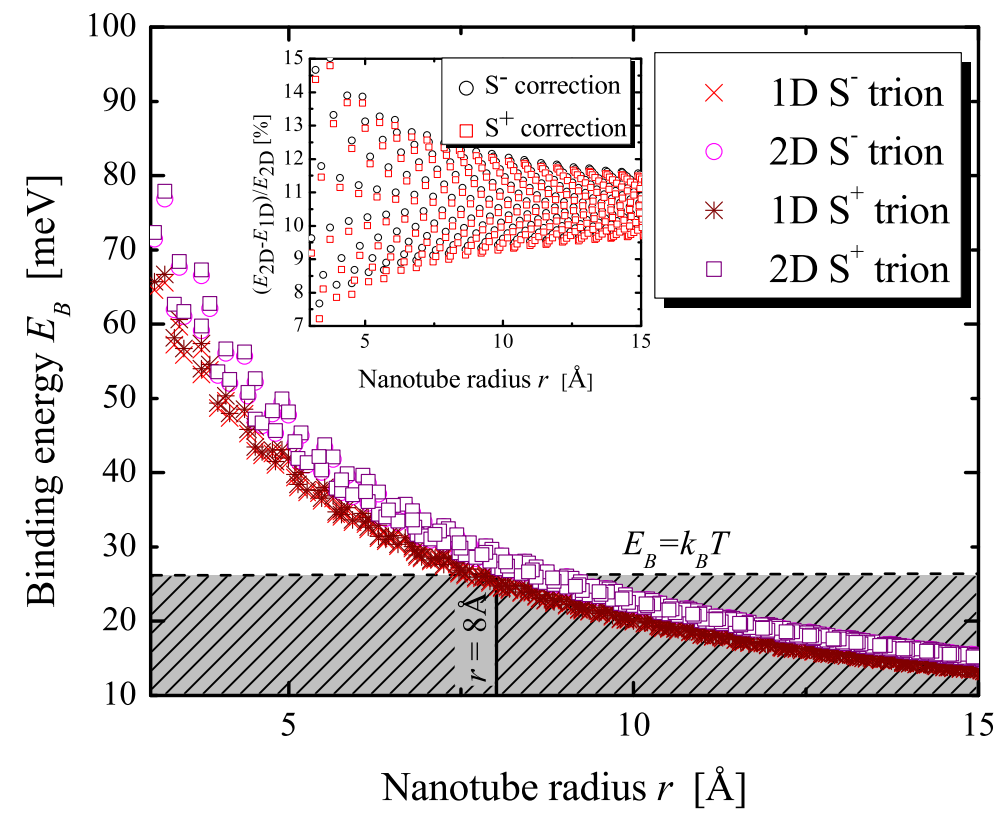

FIG. 7. Binding energy of trions in CNTs in physical units using effective electron and hole masses derived from a non-orthogonal tight binding scheme. The shaded area illustrates the instability region for which $E_{B}<k_{B} T$. Inset: percent-wise difference between the one- and two-dimensional models.

the binding energy increases with decreasing radius, CNTs with low radius constitute better candidates for observing trions. Hence, CNTs with chiral index $(6,5)$ are very promising candidates for measuring trion binding energies.

\section{CONCLUSION}

In this work, trions in CNTs have been modeled as three-particle complexes bound to the surface of a cylinder. We have shown that the angular behaviour plays a significant role with a contribution of $13 \%$ to the binding energy for cylinders with radius $r=0.10 a_{B}^{*}$. It is concluded that the energy for the $S_{0.0}^{-}$trion constitutes a fairly good approximation to both positive and negative singlet trions with mass fraction above 0.80 . We have demonstrated that the Hartree-Fock method applied to the $S_{0.0}^{-}$trion equation yields results that at best, are $60 \%$ of the correct result. We conclude that the binding energies of trions in CNTs are 
lowered with $11 \%$ on average by including the angular part of the wave function for trions.

Finally, trions are expected to be detectable in doped CNTs with $r<8 \AA$ and we consider CNTs with chiral index $(6,5)$ as a very good candidate for measuring trion binding energies.

* tfr@nanophysics.dk; http://www.nanophysics.dk

$\dagger$ tgp@nano.aau.dk

$\ddagger$ cornean@math.aau.dk

1 Y. F. Li, R. Hatakeyama, J. Shishido, T. Kato, and T. Kaneko, Appl. Phys. Lett. 90, 173127 (2007).

2 M. H. Yang, K. B. K. Teo, W. I. Milne, and D. G. Hasko, Appl. Phys. Lett. 87, 253116 (2005).

3 J. A. Misewich, R. Martel, P. Avouris, J. C. Tsang, S. Heinze, and J. Tersoff, Science 300, 783 (2003).

4 J. U. Lee, P. P. Gipp, and C. M. Heller, Appl. Phys. Lett. 85, 145 (2004).

5 J. Chen, V. Perebeinos, M. Freitag, J. Tsang, Q. Fu, J. Liu, and P. Avouris, Science 310, 1171 (2005).

6 M. S. Dresselhaus, G. Dresselhaus, R. Saito, and A. Jorio, Annu. Rev. Phys. Chem. 58, 719 (2007).

7 T. G. Pedersen, Carbon 42, 1007 (2004).

8 F. J. Teran, L. Eaves, L. Mansouri, H. Buhmann, D. K. Maude, M. Potemski, M. Henini, and G. Hill, Phys. Rev. B 71, 161309(R) (2005).

9 A. Esser, R. Zimmermann, and E. Runge, Phys. Status Solidi B 227, 317 (2001).

10 M. Semina, R. Sergeev, and R. Suris, Semiconductors 42, 1427 (2008).

11 A. S. Bracker, E. A. Stinaff, D. Gammon, M. E. Ware, J. G. Tischler, D. Park, D. Gershoni, A. V. Filinov, M. Bonitz, F. M. Peeters, and C. Riva, Phys. Rev. B 72, 035332 (2005).

12 A. Esser, E. Runge, R. Zimmermann, and W. Langbein, Phys. Rev. B 62, 8232 (2000).

13 B. Stébé and A. Ainane, Superlattices and Microstruct. 5, 545 (1989).

14 R. A. Sergeev and R. A. Suris, Nanotechnology 12, 597 (2001).

15 B. Stébé, E. Feddi, A. Ainane, and F. Dujardin, Phys. Rev. B 58, 9926 (1998).

16 T. F. Rønnow, T. G. Pedersen, and H. D. Cornean, Phys. Lett. A 373, 1478 (2009).

17 V. Perebeinos, J. Tersoff, and P. Avouris, Phys. Rev. Lett. 92, 257402 (2004). 
18 E. Chang, G. Bussi, A. Ruini, and E. Molinari, Phys. Rev. Lett. 92, 196401 (2004).

19 T. G. Pedersen, Phys. Rev. B 67, 073401 (2003).

20 J. Dahl, Introduction to the quantum world of atoms and molecules (World Scientific, Singapore, 2005).

21 T. G. Pedersen, Solid State Commun. 141, 569 (2007).

22 B. Szafran, T. Chwiej, F. M. Peeters, S. Bednarek, and J. Adamowski, Phys. Rev. B 71, 235305 (2005).

23 T. G. Pedersen and K. Pedersen, Phys. Rev. B 79, 035422 (2009).

24 A. Grüneis, C. Attaccalite, L. Wirtz, H. Shiozawa, R. Saito, T. Pichler, and A. Rubio, Phys. Rev. B 78, 205425 (2008).

25 S. Berger, F. Iglesias, P. Bonnet, C. Voisin, G. Cassabois, J. S. Lauret, C. Delalande, and P. Roussignol, J. Appl. Phys. 105, 094323 (2009).

26 Y. Ohno, S. Iwasak, Y. Murakami, S. Kishimoto, S. Maruyama, and T. Mizutani, Phys. Status Solidi B 244, 4002 (2007).

27 O. Kiowski, S. Lebedkin, F. Hennrich, S. Malik, H. Rösner, K. Arnold, C. Sürgers, and M. M. Kappes, Phys. Rev. B 75, 075421 (2007).

28 L. Landau and E. Litshitz, Electrodynamics of continuous media (Pergamen, Oxford, 1969).

29 B. Kozinsky and N. Marzari, Phys. Rev. Lett. 96, 166801 (2006).

30 T. G. Pedersen, K. Pedersen, H. D. Cornean, and P. Duclos, Nano Lett. 5, 291 (2005).

31 L. Luer, S. Hoseinkhani, D. Polli, J. Crochet, T. Hertel, and G. Lanzani, Nat. Phys. 5, 54 (2008).

32 C. Sciascia, J. Crochet, T. Hertel, and G. Lanzani, Eur. Phys. J. B(2009), doi: \bibinfo doi 10.1140/epjb/e2009-00374-1. 